\title{
Statistics of convective collapse events in the photosphere and chromosphere observed with the Hinode SOT
}

\author{
C. E. Fischer ${ }^{1}$, A. G. de Wijn ${ }^{2}$, R. Centeno ${ }^{2}$, B. W. Lites ${ }^{2}$, and C. U. Keller ${ }^{1}$ \\ 1 Utrecht University, The Netherlands \\ e-mail: [C.E.Fischer;C.U.Keller]@uu.nl \\ ${ }^{2}$ High Altitude Observatory, National Center for Atmospheric Research, Boulder, USA \\ e-mail: [dwijn;rce;lites]@ucar.edu
}

Received 7 May 2009 / Accepted 5 June 2009

\begin{abstract}
Convective collapse, a theoretically predicted process that intensifies existing weak magnetic fields in the solar atmosphere, was first directly observed in a single event by Nagata et al. (2008, ApJ, 677, L145) using the high resolution Solar Optical Telescope (SOT) of the Hinode satellite. Using the same space telescope, we observed 49 such events and present a statistical analysis of convective collapse events. Our data sets consist of high resolution time series of polarimetric spectral scans of two iron lines formed in the lower photosphere and filter images in $\mathrm{MgIb}_{2}$ and $\mathrm{CaII} \mathrm{H}$, spectral lines that are formed in the high photosphere and the lower chromosphere, respectively. We were thus able to study the implication of convective collapse events on the high photospheric and the chromospheric layers. We found that in all cases, the event was accompanied by a continuum bright point and nearly always by a brightening in the $\mathrm{Ca}$ II H images. The magnesium dopplergram exhibits a strong downflow in about three quarters of the events that took place within the field of view of the magnesium dopplergram. The physical parameters from the full Stokes profiles were obtained with the MERLIN Milne-Eddington inversion code. For each of the 49 events we determined the duration, maximum photospheric downflow, field strength increase and size. We found event durations of about $10 \mathrm{~min}$, magnetic element radii of about $0.43^{\prime \prime}$ and $0.35^{\prime \prime}$, before and after the event, respectively, and field strengths of up to $1.65 \mathrm{kG}$.
\end{abstract}

Key words. Sun: magnetic fields - Sun: photosphere - Sun: chromosphere

\section{Introduction}

Time series of solar photospheric convection as seen in the continuum at visible wavelengths and the corresponding magnetograms show a highly dynamic quiet sun. Movements, accumulation, and separation of magnetic elements are clearly determined by the granular flows (Berger \& Title 1996). Stenflo (1973) determined the field strengths of the coalesced magnetic concentrations by using the ratio between Stokes $V$ profiles of two iron lines with roughly the same excitation potential and line strength but with different effective Landé factors. This line-ratio technique revealed that the spatial-resolution-independent field strength is around $1-2 \mathrm{kG}$. This is significantly more than the equipartition field strength of $\approx 500 \mathrm{G}$ (Takeuchi 1999), which is obtained by equating the known kinetic energy density of the convective flows with the magnetic energy density. This led Parker (1978) to a possible explanation for the increase in field strength and the observed kG fields. This scenario is illustrated in cartoon form in Fig. 1.

Numerical simulations of the convective collapse process up to the destruction of the stable $\mathrm{kG}$ flux tube have been carried out in 2D and 3 D (Hasan 1985; Takeuchi 1999; Grossmann-Doerth et al. 1998; Sheminova \& Gadun 2000). The work by Grossmann-Doerth et al. (1998) suggests that, in some cases, the downward traveling mass can "bounce of" the lower layers in the atmosphere and create an upward moving flow that could lead to the destruction of the compressed flux tube. Another destruction mechanism was suggested by Sheminova \& Gadun (2000) and involved the cancellation of opposite polarity elements, destroying magnetic flux and permitting the disintegration of the flux concentration.

After years of theoretical predictions and numerical simulations, the first observational signs of the convective collapse scenario were given by Bellot Rubio et al. (2001), who studied asymmetric Stokes $V$ profiles of two photospheric, near-infrared lines at fixed spectrograph slit positions. They found redshifts in the Stokes $V$ profiles, which correspond to the downflow phase during a convective collapse process; however, shortly afterwards, they observed a decrease in the magnetic field strength, after reaching only $600 \mathrm{G}$. They also observed at the same time a strong, 3-lobed Stokes $V$ profile and argued that this is due to the appearance of an additional blueshifted Stokes $V$ profile. This can be explained by a sharp change from downflow to upflow along the line of sight and is in agreement with an upwardtraveling shock front. Bellot Rubio et al. (2001) applied an inversion code based on the thin flux tube scenario and confirmed that the profiles could be fitted very well with a discontinuity in the line of sight velocity as the result of an aborted convective collapse event. The first direct observation of a convective collapse event by Nagata et al. (2008) shows a magnetic network element undergoing a transition from a weak field strength of $400 \mathrm{G}$ to a $2 \mathrm{kG}$ flux tube. The increase of magnetic field strength was preceded by a strong downflow of $6 \mathrm{~km} \mathrm{~s}^{-1}$. They were also able to link the strong-field magnetic element to the formation of a continuum bright point. They concluded from these observations that they were witnessing a convective collapse event. We present new observations from the Hinode Spectro-Polarimeter (SP) with the addition of chromospheric intensity in $\mathrm{Ca} \mathrm{IIH}$ 

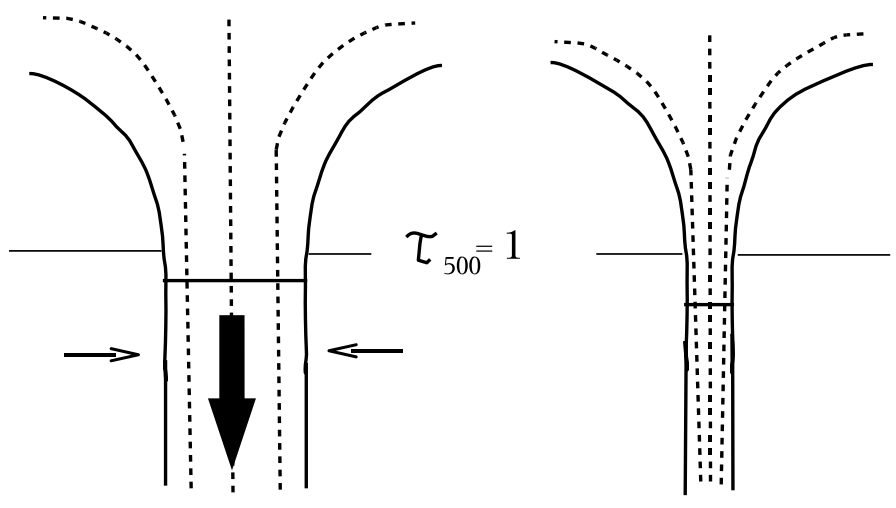

Fig. 1. Convective collapse scenario according to Parker (1978). On the left the magnetic flux tube is sitting in the intergranular lane and is strong enough to reduce convective heating, which will develop an adiabatic temperature gradient in its radiatively cooled atmosphere. The dotted lines represent the magnetic field and the horizontal line shows the $\tau=1$ level and the Wilson depression inside the flux tube. The convective collapse process assumes an initial mass downflow, represented by the big dark arrow pointing downwards. The temperature of the downwards traveling mass increases adiabatically, whilst the surrounding atmosphere shows a much steeper temperature gradient. This leads to an enhancement of the downdraft. The flux tube is then evacuated and forced to compress as the gas outside the flux tube is pressing horizontally against it, represented by the thin arrows. The limit of the compression is reached when the magnetic field pressure balances the outer gas pressure. The right side shows the compression of the flux tube, which leads to the intensification of the magnetic field strength.

and $\mathrm{MgIb}$ dopplergrams revealing the dynamics of the upper photosphere and perform a statistical analysis of 49 convective collapse events identified in several time series taken with the Hinode SOT.

\section{Data capture and processing}

We obtained 13 one-hour time sequences with the Hinode Spectro-Polarimeter (SP) each covering an area of 41 by 4.8 arcsec with a cadence of $58 \mathrm{~s}$ and a pixel size of 0.16 arcsec. The FeI $630.15 \mathrm{~nm}$ and Fe I $630.25 \mathrm{~nm}$ spectral lines were used with a spectral resolution of $0.021 \AA$. Simultaneous Narrowband Filter Imager (NFI) images in the wings of $\mathrm{Mg} \mathrm{I}_{2}$ and $\mathrm{Ca}$ II $\mathrm{H}$ filtergrams with the Broadband Filter Imager (BFI) were recorded. The $\mathrm{MgIb}_{2}$ filtergrams were taken in both the blue and red wings at $114 \mathrm{~m} \AA$ with an exposure time of $0.41 \mathrm{~s}$ and a pixel size of 0.16 arcsec. The Ca II H filtergrams were taken with an exposure time of $0.15 \mathrm{~s}$ and a pixel size of 0.11 arcsec. For more information on Hinode's Solar Optical Telescope see Kosugi et al. (2007); Suematsu et al. (2008); Shimizu et al. (2008); Ichimoto et al. (2008). We used the SolarSoft IDL routines sp_prep and fg_prep to process the data to level 1. The sp_prep procedure corrects the SP data for dark current, flat field, removes spectral and spatial drifts due to thermal instability, and merges the data from the two SP beams. The fg_prep routine corrects the filtergram (FG) data for dark current, flat field, and camera defects.

We then spatially aligned the FG and SP data. Due to the small field of view in the narrowband filter and a fixed pointing offset between FG and SP data, part of the SP field of view is outside the narrowband filter field of view. The magnesium dopplergram therefore does not cover the whole SP field of view. The FG data was resampled to the SP timing using a nearest-neighbor

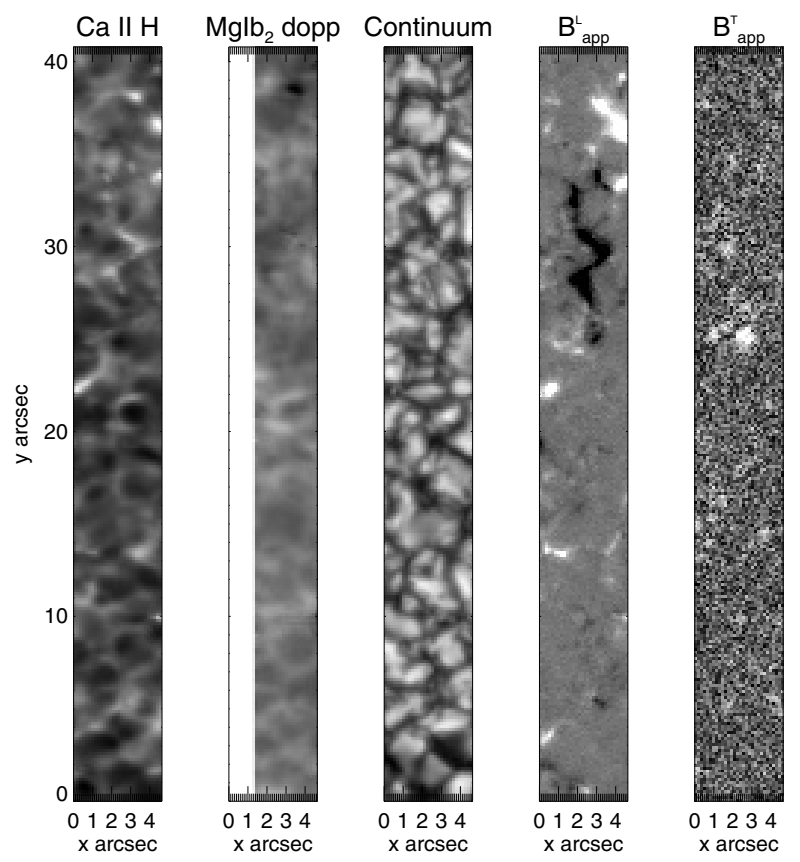

Fig. 2. Snapshot of a time sequence from the reduced data. From left to right, the coaligned images show the $\mathrm{Ca}$ II H intensity, the magnesium dopplergram, where black corresponds to downflows, the continuum intensity, the longitudinal and the transverse apparent magnetic flux (obtained by integrating over the circular and linear polarization profiles and multiplying by a known calibration constant). The left side of the $\mathrm{Mg} \mathrm{I} b_{2}$ dopplergram is cut off due to instrument misalignment and the restricted fields of view.

algorithm, with the central slit of the SP as a reference. The magnesium dopplergrams were obtained by dividing the difference between the blue wing and the red wing images by the sum. As the line-formation of the $\mathrm{MgI}_{2}$ line is quite complicated and there is no simple response height that can be assigned to the measured doppler signal (Carlsson, private communication) we could not obtain a calibration curve for the velocities in $\mathrm{km} \mathrm{s}^{-1}$ from observations or simulations. However, the formation layer of the $\mathrm{Mg} \mathrm{I} \mathrm{b}_{2}$ dopplergrams was estimated by comparing calculated $\mathrm{Mg} \mathrm{I} \mathrm{b}_{2}$ dopplergrams with snapshots from MURaM simulations (Vögler \& Schüssler 2003, see code description), and the images revealed a strong similarity in the highest photospheric layers (Vitas, private communication). In Fig. 2 we show one scan from a time sequence.

We used the Milne-Eddington inversion code MERLIN, developed by the Community Spectro-polarimetric Analysis Center $^{1}$, to retrieve the magnetic field parameters from the observed Stokes profiles (Skumanich \& Lites 1987; Lites et al. 2007).

\section{Results}

Convective collapse events were identified by eye in the observed data and the inversion parameters. Each time series was checked for sudden substantial increases in magnetic field strength preceded by a strong photospheric downflow. We found that in all these cases, the event was accompanied by a continuum bright point and nearly always by a brightening in the $\mathrm{Ca}$ II H images. The magnesium dopplergram exhibits a strong

1 see http://www. csac . hao .ucar . edu 
Table 1. Statistics of convective collapse events (see Sect. 3).

\begin{tabular}{lcccccc}
\hline \hline Type of event & $\max v$ down $\left[\mathrm{km} \mathrm{s}^{-1}\right.$ ] & start B [G] & peak B [G] & $r$ 1 [arcsec] & $r$ 2 [arcsec] & duration [min] \\
\hline 11 without Mg downflow & 3.80 & 819 & 1093 & 0.48 & 0.41 & 10.36 \\
28 with Mg downflow & 3.92 & 886 & 1356 & 0.48 & 0.34 & 10.37 \\
$10 \mathrm{Mg}$ out of FOV & 3.33 & 900 & 1262 & 0.34 & 0.31 & 9.09 \\
\hline
\end{tabular}

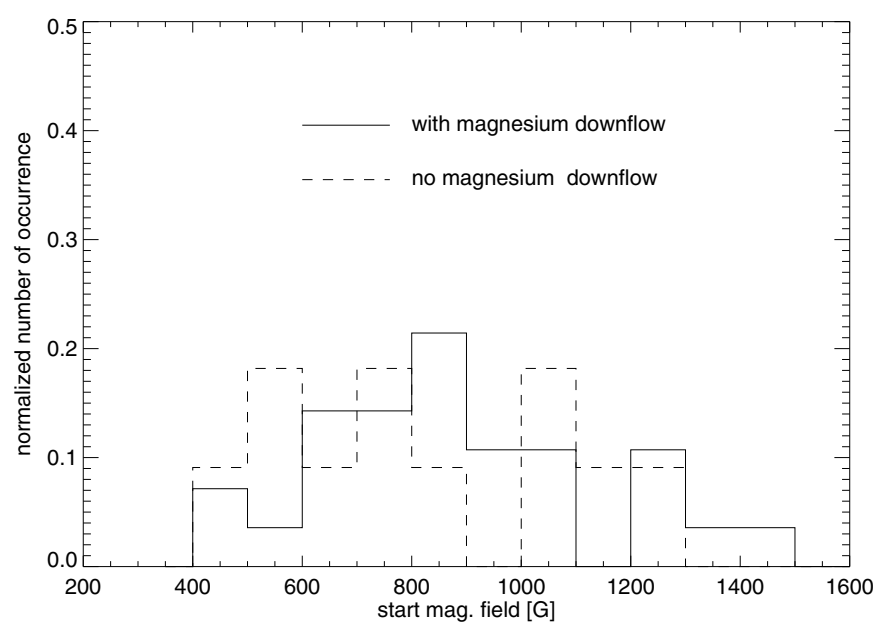

Fig. 3. Histogram of the starting values of the magnetic field strength in the magnetic elements before the convective collapse showing the number of occurrence divided by the total number of events. The dashed lines correspond to events without a downflow in the magnesium dopplergram. The solid line represents the 28 events exhibiting downflows in the magnesium dopplergram.

downflow in about three quarters of the events that took place within the field of view of the magnesium dopplergram.

Table 1 shows the statistics of the identified events in the $13 \mathrm{~h}$ of repeated $4.8 \times 41 \operatorname{arcsec}^{2}$ raster scans. To obtain the properties of the magnetic elements we followed them by eye from one time step to the next. The maximum of the line-of-sight velocity and the magnetic field strength are averages over the three highest data points, where the starting magnetic field was taken at the point of the strongest downflow. The majority of the events show equipartition field strengths at about $130 \mathrm{~s}$ before that, but it is difficult to trace the magnetic element precisely at that time. The size was determined by tracing the magnetic element in the magnetograms by eye before (radius 1) and after (radius 2) its collapse. The duration of the event was determined by taking the time when the maximum downflow occurred as the start point until after the magnetic field had reached its maximum and declined to values comparable to equipartition field strength.

The values in Table 1 are averages, which might be misleading were the distribution strongly asymmetric. The histograms of the line of sight velocity and magnetic field strength are therefore shown in Figs. 3-5. These histograms indicate that the distribution of events with downflows seen in the high photosphere are similar to those obtained from the 11 events that do not exhibit downflows.

Figure 6 shows a typical sequence in the calcium intensity, magnesium dopplergram, where black corresponds to downflows, the continuum intensity and the photospheric line of sight velocity. Clearly, a bright point can be seen in the continuum as well as in the Ca II H images forming just after a strong photospheric downflow. The magnesium dopplergram shows a strong downflow, too. The flux tube is therefore evacuated not only

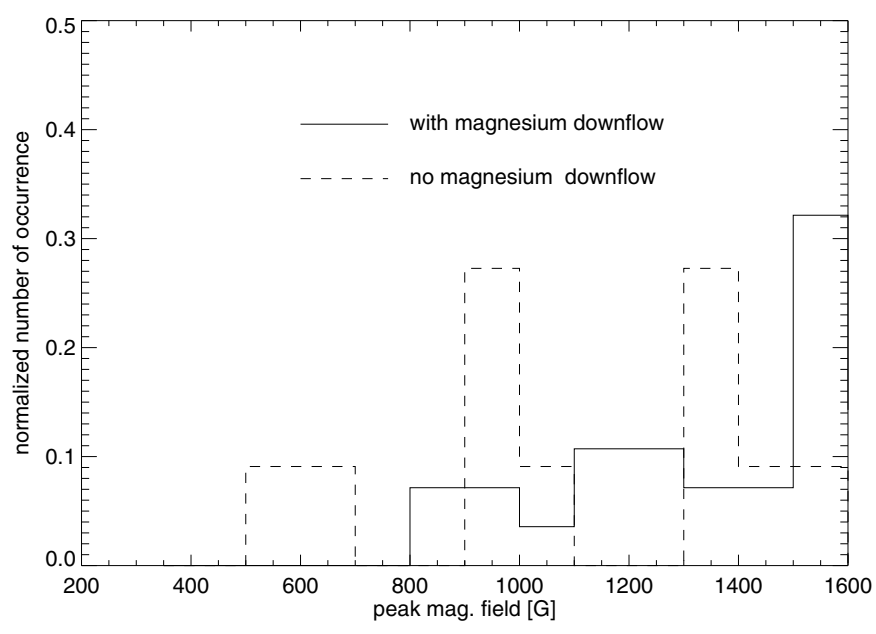

Fig. 4. Histogram of the maximum field strength reached after the intensification in the same format as Fig. 3.

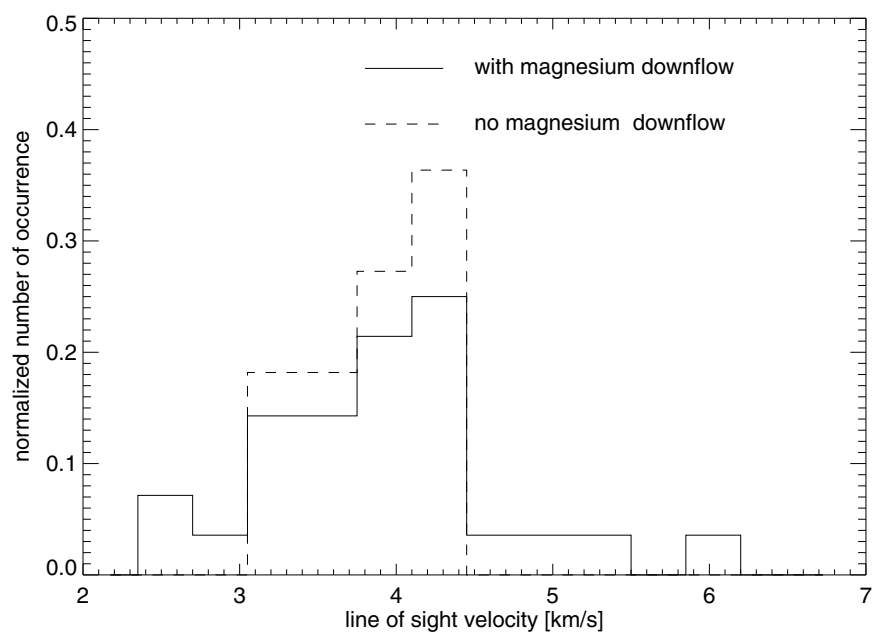

Fig. 5. Histogram of the maximum photospheric line of sight velocity in the same format as Fig. 3.

in the low photosphere but also in the high photosphere. As shown by the statistical analysis, this is the case for most events. An example of an event without significant downflows in the $\mathrm{Mg} \mathrm{I} \mathrm{b}_{2}$ dopplergram is shown in Fig. 7.

The evolution of a case with magnesium downflow signals (cf. Fig. 6) is best seen in Fig. 8, where we plot the photospheric line-of-sight velocity, magnetic field strength in the center of the magnetic element and the bright point intensity in the $\mathrm{Ca}$ II $\mathrm{H}$ filter. There seems to be an oscillatory behavior in each parameter. This is seen in most observed events and will be the subject of future research.

\section{Discussion}

As seen in Table 1, the duration of convective collapse events is about $10 \mathrm{~min}$. We can compare the duration of our events, 

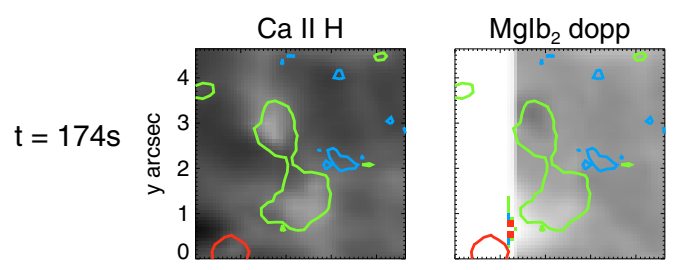

$t=464 s$
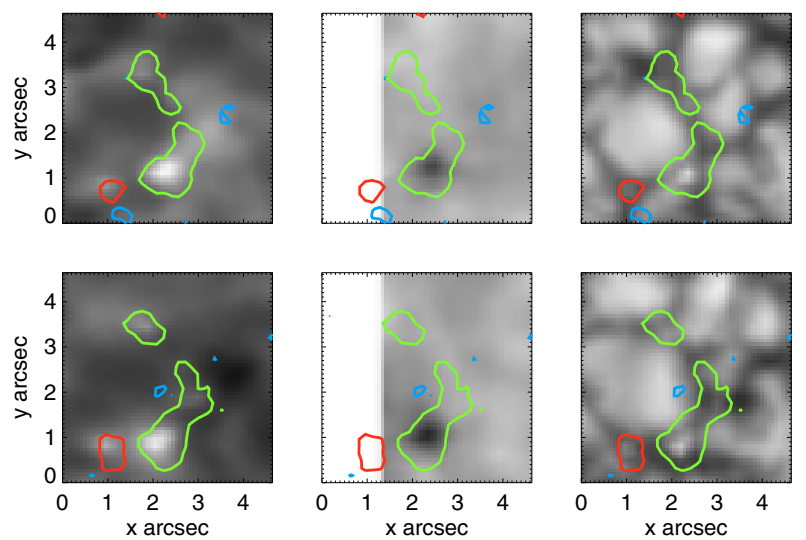

$t=580 s$
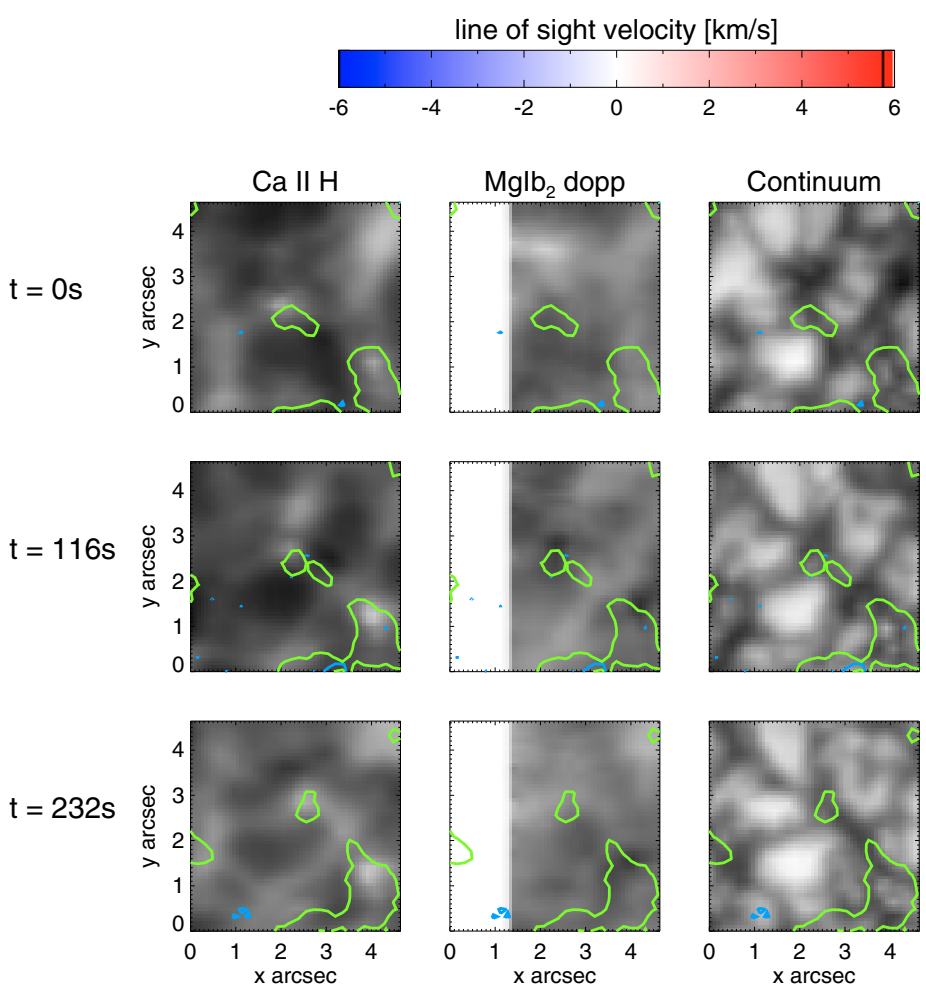

line of sight velocity $[\mathrm{km} / \mathrm{s}]$

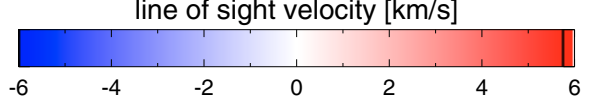

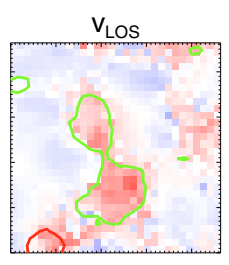
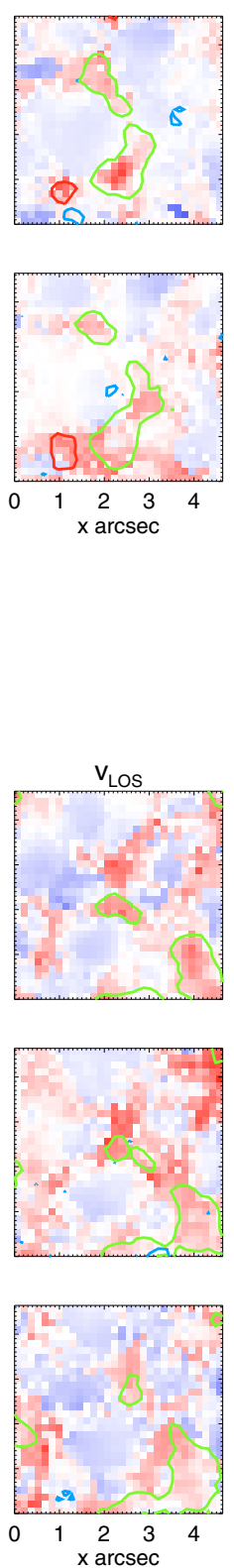

Fig. 7. A second example of a convective collapse event, in the same format as Fig. 6. This example does not show a signature in the $\mathrm{Mg} \mathrm{I} \mathrm{b}_{2}$ dopplergram.
Fig. 6. A convective collapse event associated with downflows in the upper photosphere. The field of view is $4.8^{\prime \prime}$ by $4.8^{\prime \prime}$. The columns show the $\mathrm{Ca}$ II H intensity in a linear scale, the magnesium dopplergram in arbitrary units, the continuum intensity in a linear scale, and the lineof-sight velocity derived through inversion of the Fe I line profiles, with positive values corresponding to downflows. As discussed in Sect. 2 we do not have a calibration curve for the magnesium dopplergram. The units are therefore arbitrary and black corresponds to downflows in the magnesium dopplergram. Green contours indicate the location of line-of-sight flux directed toward the observer, while red contours indicate line-of-sight flux directed away from the observer. The contour levels are at $\pm 30 \mathrm{Mx} / \mathrm{cm}^{2}$ in the apparent longitudinal flux density. The blue contours indicate the transverse apparent flux density at $140 \mathrm{Mx} / \mathrm{cm}^{2}$. i.e., the timespan from the moment of maximum downflow until the time that the field has again declined to equipartition strength, to the lifetime of magnetic elements, which has been determined by, e.g., de Wijn et al. (2008). They applied an automated feature-tracking algorithm to a six-hour sequence of magnetograms taken with the Hinode SOT, and found that magnetic elements on average have a lifetime of $10 \mathrm{~min}$. They note that magnetic elements intermittently drop below the detection limit

of the algorithm. It is tempting to speculate that the similarity between their element lifetime and our event duration results from the ability to track elements only while they are in a collapsed state.

Another process showing similar observational signatures is the emergence of magnetic flux. This process has been well analyzed by, e.g., De Pontieu (2002); Centeno et al. (2007); Martinez Gonzalez \& Bellot Rubio (2009). Centeno et al. (2007) 


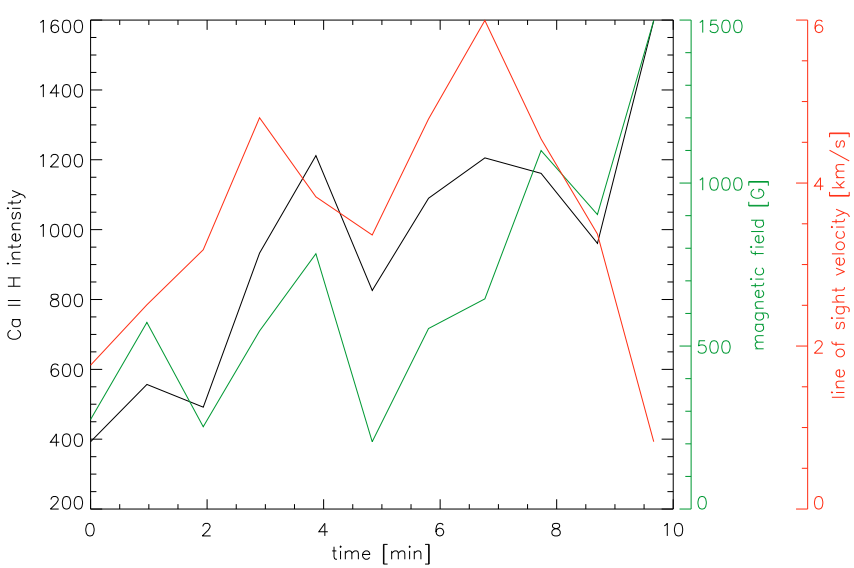

Fig. 8. Temporal development of the Ca H II intensity, the line of sight velocity and the magnetic field strength of the magnetic element from Fig. 6. Clearly, the maximum downflow takes place shortly before the increase in the $\mathrm{CaIIH}$ intensity and the magnetic field strength. The event duration in this case was longer than average, lasting about $15 \mathrm{~min}$. We show here the first $10 \mathrm{~min}$, in which the oscillatory behavior in each parameter is clearly evident.

witness two opposite polarity patches emerging within a granular structure just after a horizontal field appears. We could be observing an emergent growing magnetic field instead of the enhancement of a field by the convective collapse process. However, the difference is that the events we examine take place in the intergranular lanes, and we can observe in most cases the accumulation of flux in the intergranular lane just before the process takes place. In the event shown in Fig. 6, we can clearly see the development over minutes before the event takes place. The positive polarity flux (green contours) is carried by the granular flows and has been present in the intergranular lane during $20 \mathrm{~min}$ before the sudden increase of magnetic field. The opposite polarity patch near the observed event has been growing also through accumulation and has been also present in the intergranular lane for several minutes. It is therefore unlikely that we are observing a flux emergence event. The event in Fig. 7 does not have an opposite polarity near it before or during the collapse.

It is still unclear what mechanism causes the destruction of the strong magnetic elements. According to Bellot Rubio et al. (2001), an aborted convective collapse event can cause upward traveling shocks, which manifest themselves as asymmetries in the Stokes $V$ profiles. The events observed here could fall into this category and actually represent unfinished convective collapse events, or could be weakened or destroyed by upward flows after the collapse, as seen in simulations by Grossmann-Doerth et al. (1998). Taking a closer look at the development of the Stokes $V$ profiles (Fig. 9) of the event shown in Fig. 6, we observe a redshift caused by the downflowing material, but also strong asymmetries and 3 lobes in Stokes $V$ profiles in the center of the magnetic element. They could be signs for strong velocity gradients along the line of sight.

Figure 10 shows two examples of the observed asymmetric Stokes $V$ profiles, which can be either caused by several magnetic concentrations with different velocities in the same resolution element, or, as shown by Bellot Rubio et al. (1997), magnetic elements with strong velocity and magnetic field gradients along the line of sight. In the first case the net circular polarization (NCP) should be zero as the individual Stokes $V$ profiles are antisymmetric. In the second case there should be a substantial NCP (more than 2 percent). We determined the NCP for
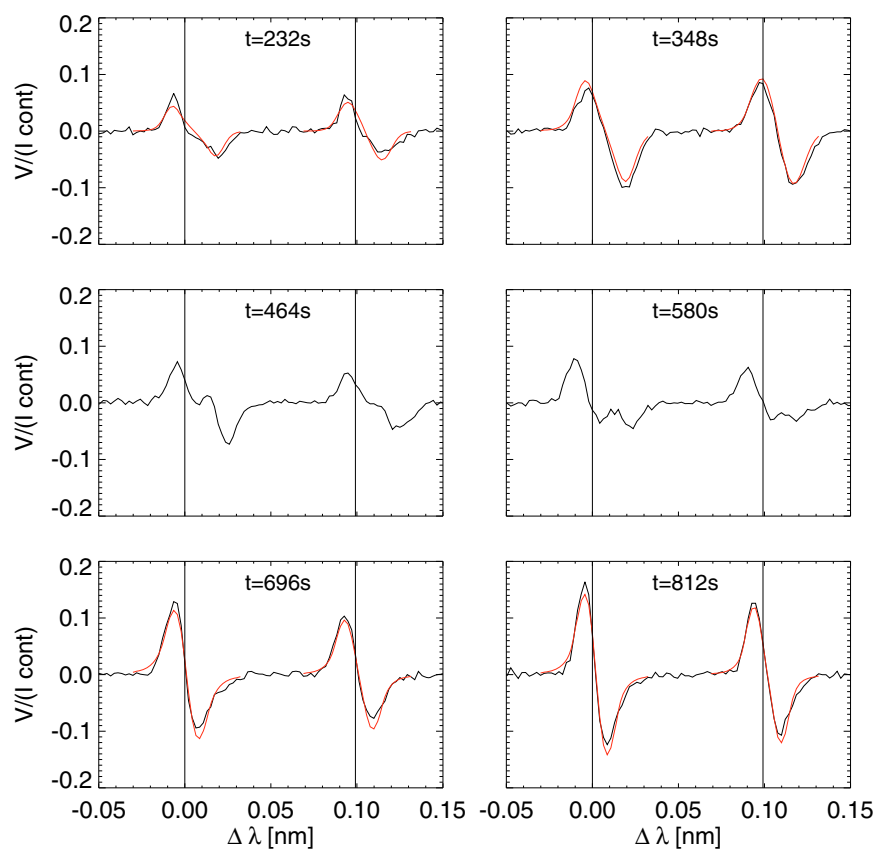

Fig. 9. Temporal evolution of the normalized Stokes $V$ profiles from the event in Fig. 6. The snapshots are $116 \mathrm{~s}$ apart and the time runs from left to right and top to bottom. The vertical lines correspond to the position of the intensity minimum averaged over the whole map. We chose the pixel at the center of the magnetic element. The strongly distorted Stokes $V$ profiles are seen after the maximal downflow and until the maximal field strength has been reached. This justifies the use of a Milne Eddington code to obtain the line of sight velocity and the starting and peak magnetic field strength, as the Stokes profiles have a normal shape at these points. We show in red the fit obtained by the inversion code for the Stokes- $V$ profiles at the beginning and end of the collapse.
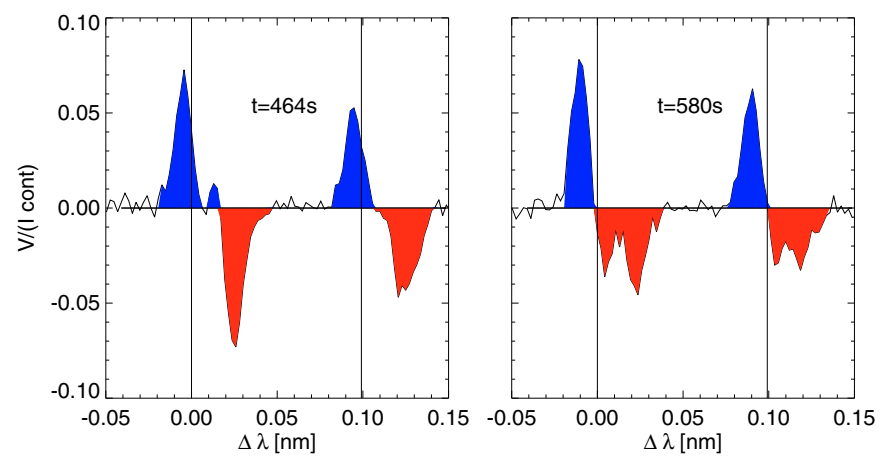

Fig. 10. Two examples of strongly distorted Stokes $V$ profiles observed within a kG magnetic element. The colors denote the blue or red lobe of the profile. We substract the blue area from the red area and divide by the sum to obtain the net circular polarization $(\mathrm{NCP})$. All profiles show a NCP of less than $1 \%$.

several asymmetric Stokes $V$ profiles, and find that all of them are around or below 1 percent. We therefore cannot confirm these events as being aborted convective collapse events or upward-traveling shocks.

However, we did find examples consistent with another process that may weaken the magnetic elements. Figure 11 shows magnetic flux of the opposite sign near to our investigated collapsed magnetic element. The flux is carried by convective motions toward the magnetic element. Then, horizontal flux is observed, and the inbound opposite polarity flux disappears almost completely. At the same time the field strength decreases sharply 


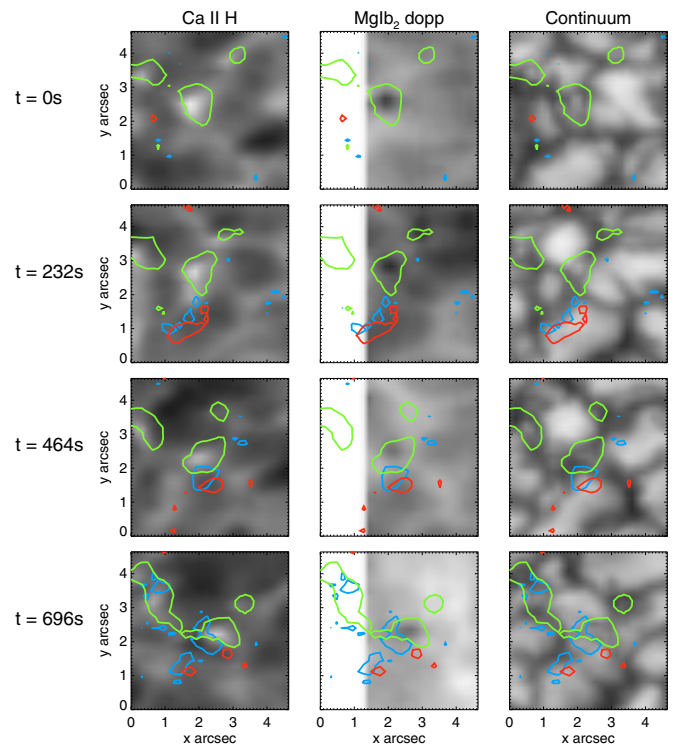

Fig. 11. Flux cancellation event leading to the weakening of the collapsed magnetic element. The figure is in the same format as Fig. 6, but without the photospheric line-of-sight velocity.

within the collapsed magnetic element. This flux cancellation scenario occurs several times in our datasets and might be the leading cause for the destruction of the newly formed $\mathrm{kG}$ fields. This is in agreement with simulations by Sheminova \& Gadun (2000), who state cancellation as the most common process of flux dispersal. As mentioned previously, we have to consider that we might actually be observing an emerging flux event. We do observe in the event of Fig. 11 opposite polarity flux emerging and growing in the granular structure in the vicinity of the observed magnetic element, but at that point the convective collapse event has already taken place and a bright point is already visible in the continuum.

The Stokes profiles were fitted using a variable filling factor. As we are in the weak field regime, the product of the filling factor and the magnetic field strength is very well determined by the inversion, but the filling factor and the magnetic field strength on their own are significantly less well determined. We therefore also computed the magnetic flux density per pixel inside the magnetic element, i.e. filling factor multiplied by the magnetic field, and averaged these values. Comparing the magnetic flux density before and after the collapse we found an increase in the vast majority of events, which is in agreement with the convective collapse scenario. The exceptions may be a consequence of the difficulty in determining the correct size of the magnetic elements by eye.

In theory, the total flux of the magnetic elements (on the order of $10^{18} \mathrm{Mx}$ ) should not be altered by the convective collapse as the increase in magnetic field strength is balanced by the decrease in area covered by the magnetic field. We do, however, find a systematic decrease in magnetic flux, which may indicate a problem with determining the flux, the field strength, the filling factor, a real decrease in flux, or a combination of all of the above.

\section{Conclusion}

We found 49 convective collapse events in the quiet solar photosphere, all showing a brightening in the continuum intensity and reaching peak magnetic field strengths of up to $1.65 \mathrm{kG}$, and with almost all events showing a brightening in the $\mathrm{Ca}$ II $\mathrm{H}$ intensity. The evacuation of the flux tube is demonstrated by downflows in the lower photosphere, which are also observed in the upper photospheric layers. Our observations provide very clear evidence of the ubiquitous occurrence of the convective collapse process. Only about a quarter of these events do not show a significant downflow in the high photosphere. We also found that the duration of the events from the downflow until the peaking and subsequent decrease of magnetic field strength is about $10 \mathrm{~min}$. In addition we confirm flux cancellation as a promising candidate for the weakening of the magnetic field strength in these strong field elements as predicted by simulations described by Sheminova \& Gadun (2000).

Acknowledgements. We thank Nikola Vitas for estimating the formation height range of the $\mathrm{Mg} \mathrm{I}_{2}$ dopplergrams and Rob Markel for providing the MERLIN inversions through the HAO inversion client. This research project has been supported by a Marie Curie Early Stage Research Training Fellowship of the European Community's Sixth Framework Programme under contract number MEST-CT-2005-020395: the USO-SP International School for Solar Physics. The National Center for Atmospheric Research is sponsored by the National Science Foundation. Alfred de Wijn and Catherine Fischer acknowledge travel support through the NCAR Early Career Scientist Assembly visitor fund. Hinode is a Japanese mission developed and launched by ISAS/JAXA, with NAOJ as domestic partner and NASA and STFC (UK) as international partners. It is operated by these agencies in co-operation with ESA and NSC (Norway).

\section{References}

Bellot Rubio, L. R., Ruiz Cobo, B., \& Collados, M. 1997, ApJ, 478, L45 Bellot Rubio, L. R., Rodríguez Hidalgo, I., Collados, M., Khomenko, E., \& Ruiz Cobo, B. 2001, ApJ, 560, 1010

Berger, T. E., \& Title, A. M. 1996, ApJ, 463, 365

Centeno, R., Socas-Navarro, H., Lites, B., et al. 2007, ApJ, 666, L137

De Pontieu, B. 2002, ApJ, 569, 474

de Wijn, A. G., Lites, B. W., Berger, T. E., et al. 2008, ApJ, 684, 1469 Grossmann-Doerth, U., Schuessler, M., \& Steiner, O. 1998, A\&A, 337, 928

Hasan, S. S. 1985, A\&A, 143, 39

Ichimoto, K., Lites, B., Elmore, D., et al. 2008, Sol. Phys., 249, 233

Kosugi, T., Matsuzaki, K., Sakao, T., et al. 2007, Sol. Phys., 243, 3

Lites, B., Casini, R., Garcia, J., \& Socas-Navarro, H. 2007, Mem. Soc. Astron. Ital., 78, 148

Martinez Gonzalez, M. J., \& Bellot Rubio, L. R. 2009, arXiv e-prints

Nagata, S., Tsuneta, S., Suematsu, Y., et al. 2008, ApJ, 677, L145

Parker, E. N. 1978, ApJ, 221, 368

Sheminova, V. A., \& Gadun, A. S. 2000, Astron. Rep., 44, 701

Shimizu, T., Nagata, S., Tsuneta, S., et al. 2008, Sol. Phys., 249, 221

Skumanich, A., \& Lites, B. W. 1987, ApJ, 322, 473

Stenflo, J. O. 1973, Sol. Phys., 32, 41

Suematsu, Y., Tsuneta, S., Ichimoto, K., et al. 2008, Sol. Phys., 249, 197

Takeuchi, A. 1999, ApJ, 522, 518

Vögler, A., \& Schüssler, M. 2003, Astron. Nachr., 324, 399 